\title{
Some numerical experiments on firn ventilation with heat transfer
}

\author{
M. R. Albert \\ U.S. Army Cold Regions Research and Engineering Laboratory, Hanover, NH 03755, U.S.A.
}

\begin{abstract}
Preliminary estimates of the thermal signature of ventilation in polar firn are obtained from two-dimensional numerical calculations. The simulations show that spatially varying surface pressure can induce airflow velocities of $10^{-5} \mathrm{~m} \mathrm{~s}^{-1}$ at $1.5 \mathrm{~m}$ depth in uniform firn, and higher velocities closer to the surface. The two-dimensional heat-transfer results generally agree with our earlier one-dimensional conclusions that the thermal effects of ventilation tend to decrease the temperature gradient in the top portions of the pack. Field observations of ventilation through temperature measurements are most likely to be observed when the firn temperature at depths on the order of $10 \mathrm{~m}$ is close to the air temperature, since steep temperature gradients can mask the thermal effects of ventilation. Preliminary indications are that, as long as surface-pressure amplitude is sufficient to move the air about in the top tens of centimeters in the snow, the resulting temperature profile during ventilation is fairly insensitive to the frequency of the surface-pressure forcing for pressure frequencies in the range $0.1-10.0 \mathrm{~Hz}$.
\end{abstract}

\section{INTRODUCTION}

The flow of air and transport of heat in snow are important processes affecting remote-sensing applications and chemical-species transport. In addition, the possibility of ventilation in polar firn has important implications for interpretation of polar ice-core records, since airflow through the firn could affect the local firn temperature and chemical processes. Field measurements of firn temperature in Greenland have been made that support the possibility of significant ventilation. Bader and others (1954) found that the near-surface snow-temperature changes were faster than could be explained by heat conduction, and attributed the differences to heat convection by moving air. More recently, Stearns and Weidner (unpublished) measured snow temperature at GISP2 in Greenland and hypothesized that ventilation may be occurring to depths of $4 \mathrm{~m}$.

There are several theoretical treatments of ventilation in the literature. Colbeck (1989) found that pressure drops created by surface topography can cause air movement in snow. Clarke and others (1987, 1991) presented one-dimensional and three-dimensional theories and showed that the near-surface turbulence can also induce airflow in firn, and that viscous-dissipation effects in the firn were insufficient to cause significant thermal disturbances. Albert and McGilvary (1992) showed that dissipation effects are several orders of magnitude lower than those of advection, and that the controlling factor in thermal effects of snow ventilation is the balance between heat conduction through the snow and heat advected by the airflow. This was demonstrated in a one-dimensional situation where air was forced through a column of snow with fixed end temperatures: the heat advected by the airflow had a significant impact on the resulting temperature distribution. For that situation the numerical results showed excellent agreement with the experimental data.

The purpose of the current investigation is to obtain preliminary estimates of the thermal signature of ventilation by conducting some two-dimensional numerical calculations. Since most of the existing field evidence of ventilation in polar firn comes from temperature sensors, it is important to examine the relationship between airflow patterns in the firn and the resulting heat balance.

\section{GOVERNING EQUATIONS}

The model employed here is a modified form of that described by Albert and McGilvary (1992). The air is treated as an incompressible medium, where conservation of mass is

$$
\frac{\partial v_{\mathrm{j}}}{\partial x_{\mathrm{j}}}=0,
$$

where $x_{\mathrm{j}}$ is a coordinate direction and $v_{\mathrm{j}}$ is the velocity of the airflow through the snow $(j=1,2$ for the current two-dimensional investigation). As described by Albert and McGilvary (1992), dissipation and compressibility heat-transfer effects are negligible, so that the assumption of incompressible flow is valid. It was also shown in that paper that vapor transport has a small effect on the heat transfer and the resulting firn-temperature profile, when compared to the more dominant control of firn 
temperature by heat conduction and by the advection of heat due to airflow. (The inverse is not true, however, since the temperature profile can have a dominant influence on the mass-transfer effects of vapor transport.) Therefore, it is safe to neglect vapor-transport effects on temperature in the current heat-transfer analysis. The airflow is assumed to follow Darcy's law, where the Darcy velocity, $v_{\mathrm{j}}$, is given by

$$
v_{\mathrm{j}}=-\frac{k_{\mathrm{jk}}}{\mu}\left[\frac{\partial P}{\partial x_{\mathrm{k}}}\right],
$$

where $P$ is pressure, $k_{\mathrm{jk}}$ is the permeability tensor, and $\mu$ is the viscosity of the air. Repeated indices imply summation. Equations (1) and (2) are combined to give Equation (3) for airflow:

$$
\frac{\partial}{\partial x_{\mathrm{j}}}\left[-\frac{k_{\mathrm{jk}}}{\mu}\left[\frac{\partial P}{\partial x_{\mathrm{k}}}\right]\right]=0 .
$$

The heat transfer is described by the advection-diffusion equation:

$$
\left(\rho C_{\mathrm{p}}\right)_{\mathrm{s}} \frac{\partial T}{\partial t}+\phi \rho_{\mathrm{a}_{0}} C_{\mathrm{p}_{\mathrm{a}}} v_{\mathrm{j}} \frac{\partial T}{\partial x_{\mathrm{j}}}=-\frac{\partial}{\partial x_{\mathrm{j}}}\left[\lambda_{\mathrm{jk}} \frac{\partial T}{\partial x_{\mathrm{k}}}\right],
$$

where

$$
\left(\rho C_{\mathrm{p}}\right)_{\mathrm{s}}=\phi\left(\rho C_{\mathrm{p}}\right)_{\mathrm{a}}+(1-\phi)\left(\rho C_{\mathrm{p}}\right)_{\mathrm{i}} .
$$

In these equations $T$ is temperature, $C_{\mathrm{p}}$ is specific heat, $\lambda_{\mathrm{jk}}$ is the thermal conductivity tensor, $\rho$ is density, $\phi$ is porosity, the subscripts s, a, and i refer to snow, air, and ice, respectively, and repeated indices $\mathrm{j}$ and $\mathrm{k}$ imply summation.

Equations (3) and (4) are solved numerically using a finite element model with linear triangular elements developed by W. R. McGilvary and M. R. Albert at the Cold Regions Research and Engineering Laboratory. The simulations described below will focus on the effect of the two-dimensional airflow velocity field within the firn on the temperature distribution there. The airflow in the firn will be driven by surface-pressure effects. The temperature distribution will include the effects of fixed surface temperatures and advective effects due to air motion within the firn.

\section{NUMERICAL SIMULATIONS}

In the one-dimensional analysis, Albert and McGilvary (1992) showed that airflow through a cylindrical snow sample with fixed temperatures at the inlet and outlet could have very pronounced effects on the temperature regime, with the ventilation effect of decreasing the temperature gradients near the inlet and increasing them near the outlet. In that case, air (saturated with vapor at the inlet) was forced through a cylinder of snow, where the inlet temperature was maintained at a lower temperature than the temperature at the outlet. It was also shown that a strong overall temperature gradient (imposed by the thermal boundary conditions) could drive the heat conduction enough to counteract the thermal effects of ventilation. Thus cases with significant airflow through snow may not be detectable from temperature measurements. In two and three dimen- sions, the airflow patterns are much more complicated than in the one-dimensional case. It is of interest now to investigate whether the multidimensional thermal signature is similar to the one-dimensional case; i.e., whether ventilation decreases temperature gradients near the surface and increases them deeper in the firn.

The simulations consider a segment of firn $6 \mathrm{~m}$ long and $6 \mathrm{~m}$ deep. For these estimates, the firn surface profile is assumed to be flat, with various forms of induced pressure fluctuations on that surface. A finite-element mesh with 1056 triangular elements, becoming successively finer mesh from the bottom to the top surface, was employed for the simulations. In each of the following simulations, the region is assumed to be at a uniform initial temperature.

The case considered is the simplest possible case: homogeneous firn. The density is $300 \mathrm{~kg} \mathrm{~m}^{-3}$ and grain size $1 \mathrm{~mm}$, which gives a permeability equal to $7 \mathrm{x}$ $10^{-9} \mathrm{~m}^{2}$ (Shimuzu, 1970), and thermal conductivity $0.25 \mathrm{~W} \mathrm{~m}^{-1} \mathrm{~K}$ (Mellor, 1977). For this application, the permeability and thermal conductivity are taken to be uniform throughout the domain. The initial temperature is $-30^{\circ} \mathrm{C}$ throughout, with surface step change in temperature to $-25^{\circ} \mathrm{C}$.

The first simulation imposes a steady-state, sinusoidal pressure of the form $P=A \sin \left(2 \pi x_{1} / L\right)$, with $A=10 \mathrm{~Pa}$ and $L=1.09 \mathrm{~m}$ across the top boundary, as depicted in Figure 1. This peak amplitude pressure of $10 \mathrm{~Pa}$ is taken from measurements conducted by Farrell and others (1966), who put an array of pressure transducers over a stretch of soil with short $(3 \mathrm{~cm})$ grass, and found that the amplitude of the difference in horizontal pressure measurements increases with the spacing of the measurements. The resulting steady-state isotherms are shown in Figure 2a. It should be noted that the dips in temperature near the side boundaries result from the use of a zerogradient boundary condition on the pressure solution used there for convenience; the center $3 \mathrm{~m}$ is the primary region of interest. Simulations were run with a denser grid and with wider $x_{1}$-dimensions, but the temperatures in the center $3 \mathrm{~m}$ were the same as depicted here. For comparison, the isotherms resulting from heat conduction

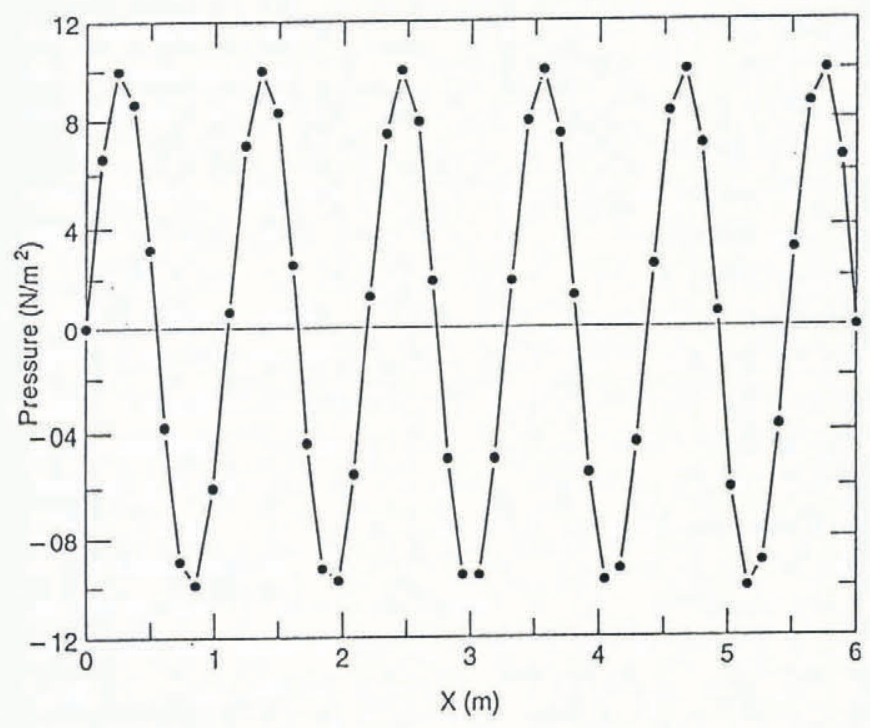

Fig. 1. Pressure forcing on the surface of the firn. 

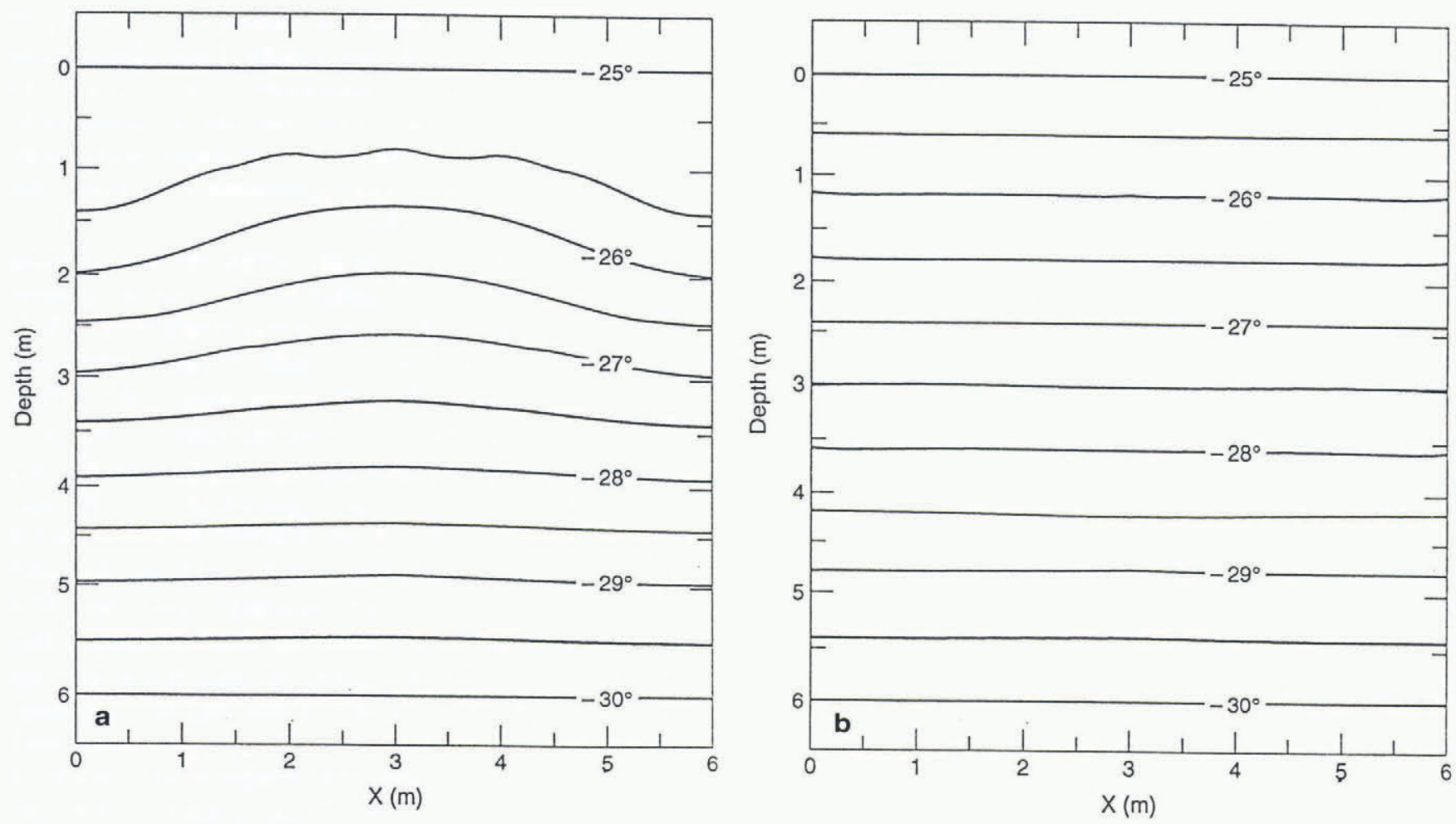

Fig. 2. Isotherms resulting from: a, pressure amplitude of $10 \mathrm{~Pa}$ and an overall temperature gradient of $0.83^{\circ} \mathrm{Cm}^{-1} ; \mathrm{b}$, heat conduction alone, without ventilation, for an overall (top-to-bottom) temperature gradient of $0.83^{\circ} \mathrm{Cm}^{-1}$.

without ventilation are shown in Figure $2 \mathrm{~b}$. Comparison of Figure $2 \mathrm{a}$ and $2 \mathrm{~b}$ shows that ventilation does tend to unify the surface temperatures in the top tens of centimeters of the snow, decreasing the temperature gradient there, as was also true in the one-dimensional case. The increase in temperature gradient at lower depth is much less significant than in the one-dimensional case, however.

The airflow velocity field is two-dimensional and thus each velocity vector has two components, but it is the scalar magnitude of the resultant velocity vector at each location that is important in chemical reactions. In Figure 3 the magnitudes of the resultant velocity vectors are plotted for $x_{1}$ locations at five selected depths. The magnitude of the velocity varies from approximately $0.02 \mathrm{~m} \mathrm{~s}^{-1}$ at the very top of the firn, to $10^{-3} \mathrm{~m} \mathrm{~s}^{-1}$ at $0.6 \mathrm{~m}$ depth, to near $10^{-5} \mathrm{~m} \mathrm{~s}^{-1}$ at $1.5 \mathrm{~m}$ depth.

For an indication of the effect of the overall

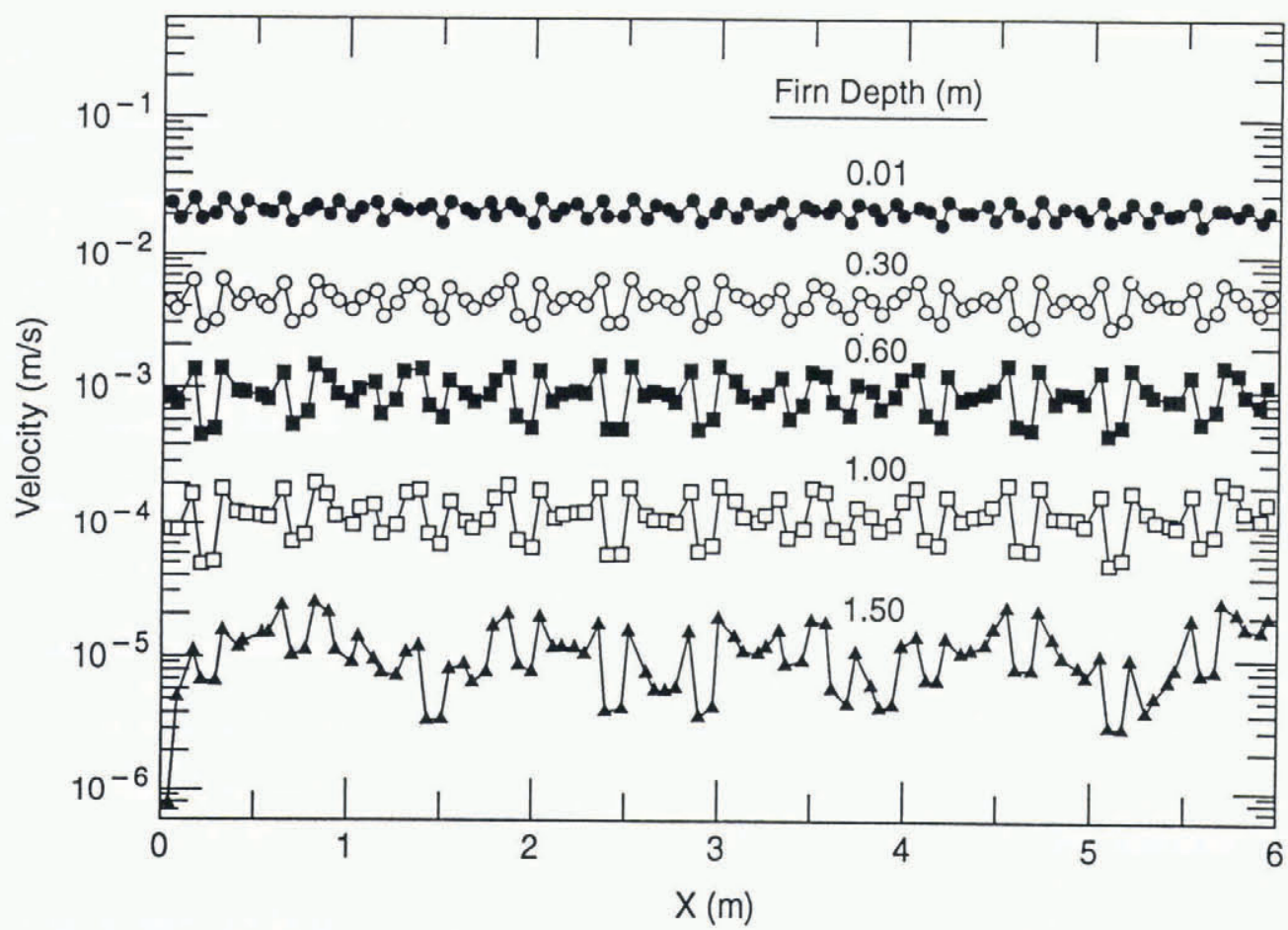

Fig. 3. Magnitudes of the resultant airflow velocity vectors at five selected depths in the firn, for a surface-pressure amplitude of $10 \mathrm{~Pa}$. 


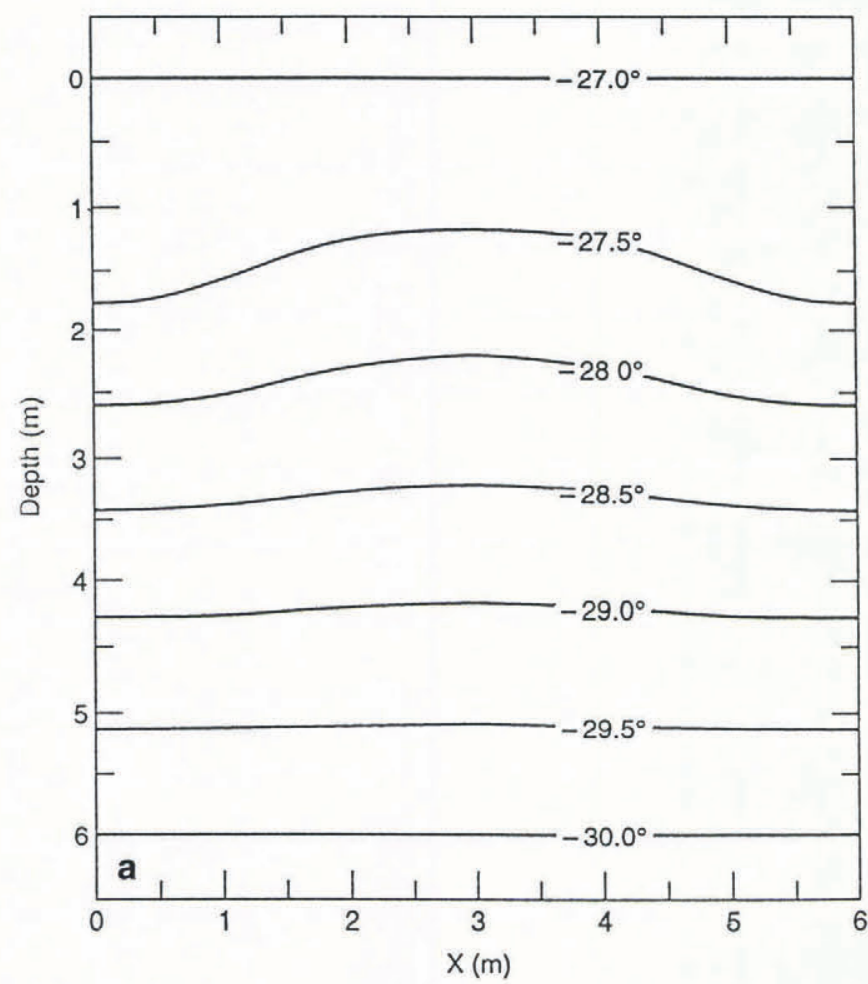

thermal signatures, creating near-isothermal temperature profiles in the top regions, are similar. Field observations of ventilation through temperature measurements are most likely to be observed when the firn temperature at depths near 6-10 $\mathrm{m}$ are close to the air temperature, since steeper overall temperature gradients can mask the thermal effects of ventilation.

Clearly the amplitude of the surface-pressure forcing will influence the airflow velocities. For the same snow properties as above, and surface temperature of $-25^{\circ} \mathrm{C}$, the simulation is run with a peak pressure amplitude difference equal to $1.0 \mathrm{~Pa}$. The magnitudes of the airflow velocities are one-tenth of those calculated with peak amplitude $10.0 \mathrm{~Pa}$, so that the flow velocity in the top several millimeters is approximately $0.002 \mathrm{~m} \mathrm{~s}^{-1}$, decreasing to a flow of $10^{-4} \mathrm{~m} \mathrm{~s}^{-1}$ at a depth of $0.6 \mathrm{~m}$, still a significant magnitude when compared to diffusive transfer mechanisms in nonconvective processes. This reduction in flow velocity from the first case means that conductive heat-transfer processes may dominate the temperature profile. In Figure 5 the resulting temperature distribution is shown. Indeed, by comparison with Figure $2 b$, we see that the temperature profile is practically the same as that of pure conduction with no ventilation; in this case the thermal effects of conduction are able to overwhelm those of advection.

For all of the above simulations, the surface-pressure forcing varies over distance but not in time. In nature the surface-pressure forcing imposed by turbulent winds will be variable in both space and time. While a sinusoidal wave train representation is a simplification, it is useful to investigate the thermal effects for several frequencies in time of surface-pressure forcing. The final simulation

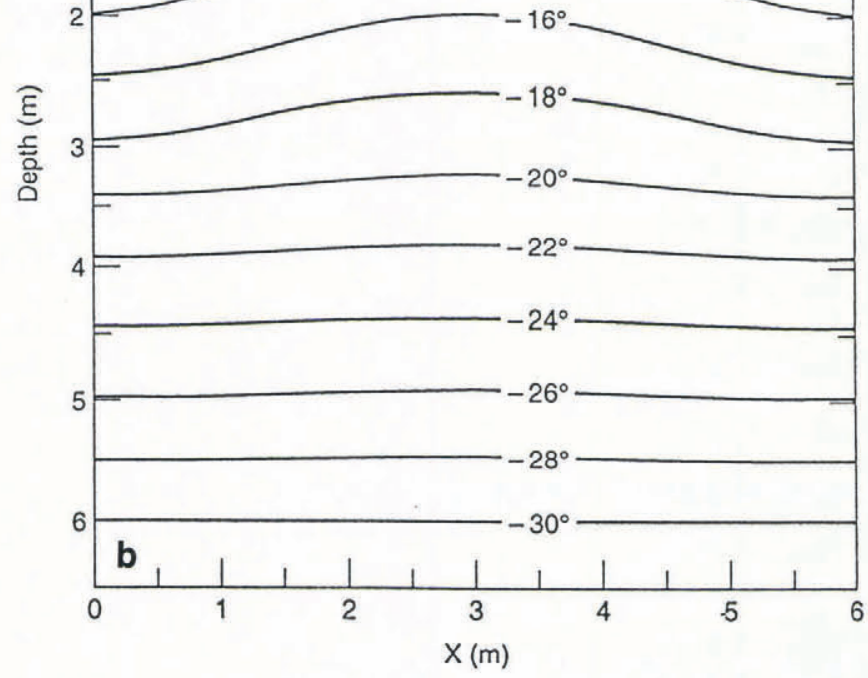

Fig. 4. Isotherms for surface-pressure amplitude of $10 \mathrm{~Pa}$. $a$, overall temperature gradient of $0.50^{\circ} \mathrm{C} \mathrm{m}^{-1} ; b$, overall temperature gradient of $3.3^{\circ} \mathrm{C} \mathrm{m}^{-1}$.

temperature gradient on the temperature distribution with this same airflow regime, the resulting temperature regimes are plotted in Figure $4 \mathrm{a}$ and $4 \mathrm{~b}$ for the same snow but with surface temperatures of $-27^{\circ}$ and $-10^{\circ} \mathrm{C}$, respectively. It is evident that the more severe the overall temperature gradient, the more obscure are the thermal effects of ventilation, becoming confined to the surface portions of the pack. Thus, although the airflow patterns in two dimensions are much different from those in the one-dimensional simulations, the general trends of

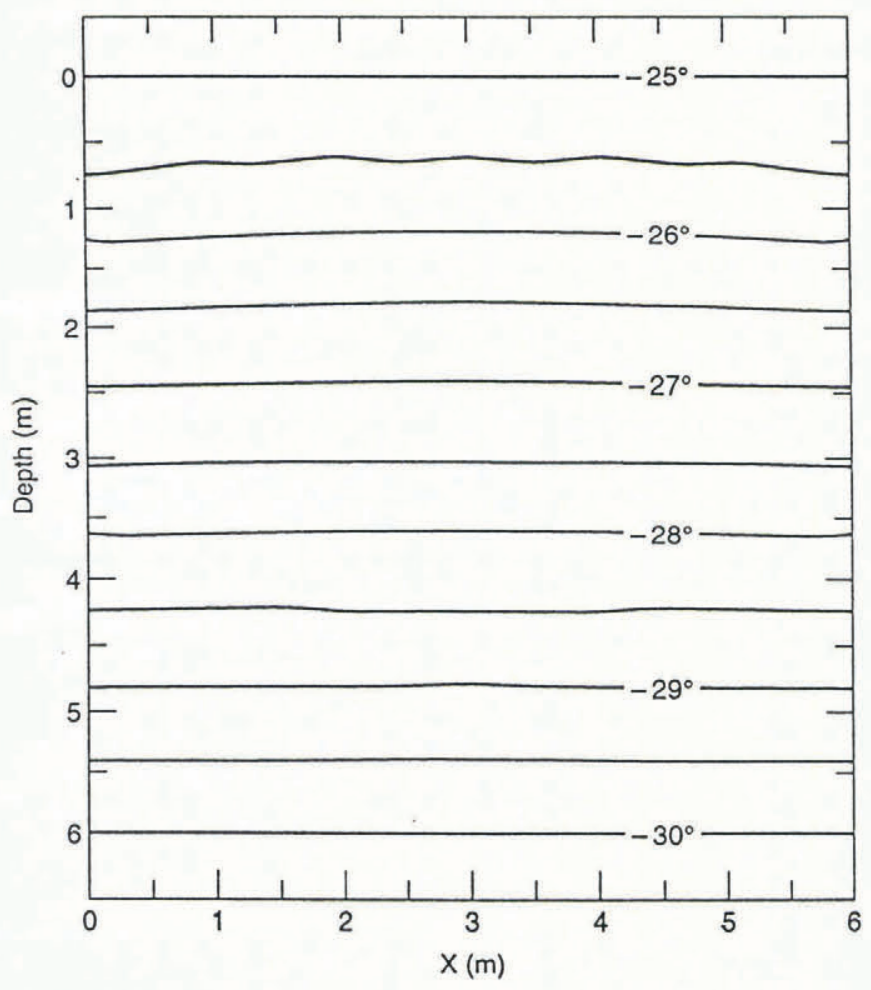

Fig. 5. Isotherms resulting from a surface-pressure amplitude of $1.0 \mathrm{~Pa}$ and overall temperature gradient of $0.83^{\circ} \mathrm{C} \mathrm{m}^{-1}$. 


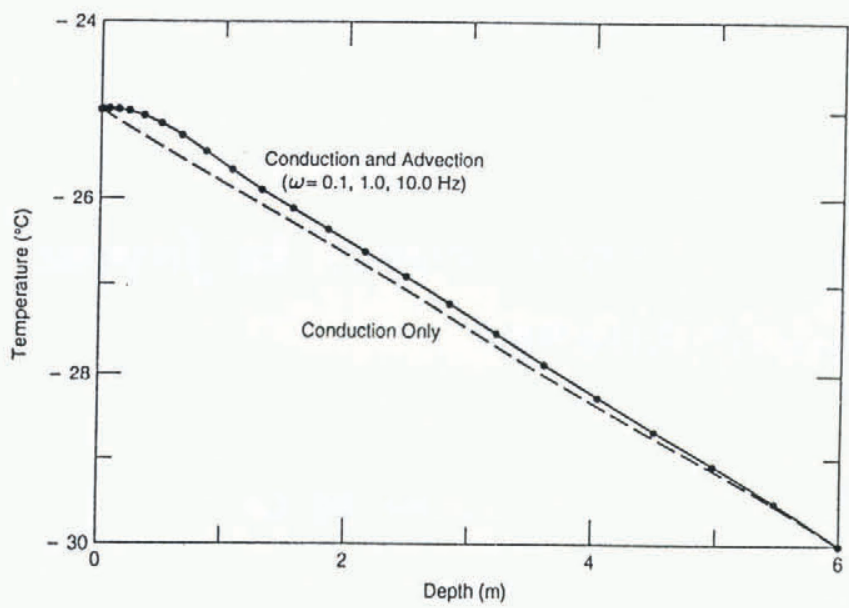

Fig. 6. Vertical temperature profile at $x_{1}$ location $3 \mathrm{~m}$, for a surface-pressure amplitude $10 \mathrm{~Pa}$, surface-pressure frequencies $0.1,1.0$ and $10.0 \mathrm{~Hz}$, and an overall temperature gradient $0.83^{\circ} \mathrm{C} \mathrm{m}^{-1}$. Also plotted for comparison is the linear, steady heat-conduction profile.

assigns a surface pressure that varies both in space and in time according to

$$
P=A \sin \left(2 \pi \frac{x}{L}+\omega t\right)
$$

where $A=10 \mathrm{~Pa}, \omega$ is frequency, and $t$ is time (in s). In Figure 6 the vertical temperature profile in the center $\left(x_{1}=3 \mathrm{~m}\right)$ of the domain at time $t=1 \mathrm{~h}$ is plotted for surface pressure frequencies of $\omega=0.1,1.0$, and $10.0 \mathrm{~Hz}$, along with the temperature profile that would result from heat conduction alone (without ventilation). Several points are evident. First, varying the frequency in time of the surface-pressure forcing between 0.1 and $10.0 \mathrm{~Hz}$ had no effect on the resulting temperature distribution after one hour of simulated time. This may be an indication that the temperature profile during ventilation is fairly insensitive to the frequency of the pressure forcing, as long as there is sufficient surface-pressure amplitude to move the air about in the top decimeters of the snow. A more exhaustive study of this preliminary result is forthcoming.

Secondly, for this overall temperature gradient $\left(0.83^{\circ} \mathrm{Cm}^{-1}\right)$, the local temperature gradient was most affected by ventilation in the top meter of firn. Below the top meter, the temperature shift is dominated by heat conduction of the temperature changes induced in the top meter. We see that, although ventilation from surfacepressure forcing induces significant airflow primarily in the top several meters of firn, the temperature is nevertheless altered at deeper locations by conduction, because of the airflow-induced temperature changes within the top meter.

\section{CONCLUSIONS}

Numerical simulations of two-dimensional ventilation and heat transfer in a uniform firn show that spatially varying surface pressure can induce airflow velocities of $10^{-5} \mathrm{~m} \mathrm{~s}^{-1}$ at $1.5 \mathrm{~m}$ depth, and at higher velocities closer to the surface. The heat transfer results generally agree with earlier one-dimensional conclusions (Albert and McGilvary, 1992) that the thermal effects of ventilation are to decrease the temperature gradient in the top portions of the pack, and that the depth to which the temperature gradient is changed by ventilation depends on the flow velocity and also on the overall temperature gradient. Field observations of ventilation through temperature measurements are most likely to be observed when the firn temperature at depths on the order of $10 \mathrm{~m}$ are close to the air temperature, since steep temperature gradients can mask the thermal effects of ventilation. Preliminary indications are that the temperature profile during ventilation is fairly insensitive to the frequency of the pressure forcing for pressures with a frequency range 0.1$10.0 \mathrm{~Hz}$, as long as there is sufficient surface-pressure amplitude to move the air about in the top tens of centimeters of the snow.

This investigation focused on uniform firn, while in nature, polar firn is layered. Ongoing work will focus on effects of layering and surface relief on ventilation processes, and will expand the analysis to include vapor and chemical-species transport.

\section{ACKNOWLEDGEMENTS}

I wish to thank Randy McGilvary for assistance in implementing the pressure boundary conditions in the code, and thank Sam Colbeck, Bert Davis, and anonymous reviewers for their technical reviews. This work was funded by U.S. Army work unit 4A161102 AT24 SS-E09, Cold Regions Surface-Air Boundary Transfer Processes.

\section{REFERENCES}

Albert, M. R. and W.R. McGilvary. 1992. Thermal effects due to airflow and vapor transport in dry snow. f. Glaciol., 38(129), 273-281.

Bader, H., R. Haefeli, E. Bucher, J. Neher, O. Eckel and C. Thams. 1954. Snow and its metamorphism. SIPRE Translation 14.

Clarke, G. K. C. and E. D. Waddington. 1991. A threedimensional theory of wind pumping. F. Glaciol., 37 (125), 89-96.

Clarke, G. K. C., D. A. Fisher and E. D. Waddington. 1987. Wind pumping: a potentially significant heat source in ice sheets. International Association of Hydrological Sciences Publication 170 (Symposium at Vancouver 1987 - The Physical Basis of Ice Sheet Modelling), 169-180.

Colbeck, S. C. 1989. Air movement in snow due to windpumping. 7. Glaciol., 35(120), 209-213.

Farrell, D. A., E. G. Greacen and C. G. Gurr. 1966. Vapor transfer in soil due to air turbulence. Soil Sci., 102(5), 305-313.

Mellor, M. 1977. Engineering properties of snow. $\mathcal{J}$. Glaciol., 19(81), 15-66.

Shimizu, H. 1970. Air permeability of deposited snow. Contrib. Inst. Low Temp. Sci., Ser. A 22.

Stearns, C. R. and G. A. Weidner. Unpublished. Report on the 1991 season on the Greenland crest. Draft report 1991. University of Wisconsin - Madison.

The accuracy of references in the text and in this list is the responsibility of the author, to whom queries should be addressed. 